\title{
Promotion of Oral Anticoagulation in Patients with Atrial Fibrillation: Absolute Impact of Interventions attained in the IMPACT-AF trial
}

\author{
Cyrus R Kumana, Bernard M Cheung
}

Medicine, The Unversity of Hong Kong, Hong Kong

Background: The under use of oral anticoagulants (OACs) in patients with atrial fibrillation (AF) is well known. The recently published IMPACT-AF trial [1] suggests that certain interventions can promote their appropriate use. This prospective, international, cluster-randomized, controlled trial compared the impact of on-going, multifaceted, educational interventions versus usual-care for up to 1 year in patients with AF. However, the value of such resourceintensive efforts to attain the benefits described must be gauged in absolute as well as relative terms.

Methods: Based on the published results of the IMPACT-AF trial, we derived the apparent 6-month and 1-year unadjusted: i) Odds Ratios, and ii) absolute success rates, (expressed as Number Needed to Target or 'NNTar' to achieve one success) for promoting oral anticoagulant. These parameters and their 95\% CIs were determined for all recruited intervention and control group AF patients (1187 \& 1094, respectively) and for those not using them at baseline (380 \& 389 , respectively). All calculations were based on previously reported algorithms to derive relative risk (RR) and number needed to treat (NNT). [2]

Results: Calculated parameters for respective intervention group patients starting oral OACs and those not taking them at baseline are shown in the table.

Conclusions: The NNTar values after 1 year of ongoing educational interventions appear impressive compared to NNT/year values (e.g. 63-256) encountered in many drug treatment trials. [2] Nevertheless, decisions to deploy such resource-intensive interventions must depend on local resource implications and priorities, together with locally anticipated hard end-point benefits from embolic event prevention and safety issues.

References: [1] Vinereanu D et al 2017, Lancet 390:1737-46. [2] Kumana CR et al 1999, JAMA 282:1899-901. 